\title{
Joint 3-D Positioning and Power Allocation for UAV Relay Aided by Geographic Information
}

\author{
Pengfei Yi, Liang Zhu, Lipeng Zhu, Student Member, IEEE, Zhenyu Xiao, Senior Member, IEEE, Zhu \\ Han, Fellow, IEEE, and Xiang-Gen Xia, Fellow, IEEE
}

\begin{abstract}
In this paper, we study to employ geographic information to address the blockage problem of air-to-ground links between UAV and terrestrial nodes. In particular, a UAV relay is deployed to establish communication links from a ground base station to multiple ground users. To improve communication capacity, we first model the blockage effect caused by buildings according to the three-dimensional (3-D) geographic information. Then, an optimization problem is formulated to maximize the minimum capacity among users by jointly optimizing the 3$D$ position and power allocation of the UAV relay, under the constraints of link capacity, maximum transmit power, and blockage. To solve this complex non-convex problem, a twoloop optimization framework is developed based on Lagrangian relaxation. The outer-loop aims to obtain proper Lagrangian multipliers to ensure the solution of the Lagrangian problem converge to the tightest upper bound on the original problem. The inner-loop solves the Lagrangian problem by applying the block coordinate descent (BCD) and successive convex approximation (SCA) techniques, where UAV 3-D positioning and power allocation are alternately optimized in each iteration. Simulation results confirm that the proposed solution significantly outperforms two benchmark schemes and achieves a performance close to the upper bound on the UAV relay system.
\end{abstract}

Index Terms-UAV, relay communications, geographic information, 3-D positioning, power allocation.

\section{INTRODUCTION}

$\mathbf{U}$ NMANNED aerial vehicles (UAVs) have attracted increasing attention for enhancing the performance of wireless communication networks [1]-[6]. Compared to conventional cellular systems, UAV-assisted communications do not rely on fixed terrestrial infrastructures, and can be flexibly deployed over a target area in an on-demand and cost-effective manner. For instance, UAVs may serve as aerial base stations (BSs) or relays to support ground user equipments (UEs), as

This work was supported in part by the National Key Research and Development Program (Grant No. 2020YFB1806800), the Defense Industrial Technology Development Program, and the National Natural Science Foundation of China (NSFC) under grant numbers 61827901 and 91738301. The corresponding author is Dr. Zhenyu Xiao with Email xiaozy@buaa.edu.cn.

Pengfei Yi, Lipeng Zhu, Zhenyu Xiao are with the School of Electronic and Information Engineering, Beihang University, Beijing 100191, China. (yipengfei@ buaa.edu.cn, zhulipeng@buaa.edu.cn, xiaozy@buaa.edu.cn)

Liang Zhu is with the IoT Division of CTTL-Terminals Laboratory, China Academy of Information and Communication Technology, Beijing 100191, China. (zhuliang@caict.ac.cn)

Zhu Han is with the Department of Electrical and Computer Engineering in the University of Houston, Houston, TX 77004 USA, and also with the Department of Computer Science and Engineering, Kyung Hee University, Seoul, South Korea, 446-701. ( zhan2@uh.edu).

Xiang-Gen $\mathrm{Xia}$ is with the Department of Electrical and Computer Engineering, University of Delaware, Newark, DE 19716, USA. (xianggen@udel.edu) well as extend communication coverage in hot-spot regions or disaster areas.

Benefiting from their three-dimensional (3-D) mobility, UAVs may adjust their positions or trajectories according to traffic demands to provide satisfactory communication service. As a result, a large number of works have been devoted to UAV-assisted communication systems [7]-[17]. For instance, in [7], two-dimensional (2-D) trajectory, multiuser scheduling and association, and transmit power were jointly designed for a downlink multi-UAV enabled communication system, aiming to maximize the minimum throughout among all ground UEs. In [8], 2-D UAV trajectory and transmit beamforming vector were jointly optimized to minimize the total power consumption for multiuser downlink multiple-input single-output (MISO) UAV communication systems. UAV jittering, UE location uncertainty, wind speed uncertainty, and no-fly zones were taken into account to provide reliable communication services. In [9], UAV positioning, analog beamforming, and power control were jointly optimized for a millimeter-wave full-duplex UAV relay communication system. A secure communication problem was investigated for a UAV-enabled communication system in [10], where UAV trajectory and UE scheduling were jointly designed for maximizing the minimum secrecy rate. In [13], UAV trajectory and transmit power were jointly designed to achieve efficient spectrum sharing for a full-duplex UAV relaying system with underlaid device-to-device (D2D) communications. A UAV-enabled energy-efficient Internet of Things (IoT) communication system was considered in [14], where 3-D placement and resource allocation of multiple UAVmounted BSs were optimized to minimize the transmit power of IoT devices. In [16], 3-D positions of UAVs, UE clustering, and frequency band allocation were optimized to improve the coverage rate as well as to minimize the number of required UAVs for a multiple UAV-BSs assisted communication system.

To make the problem more tractable, the aforementioned works mostly employ simplified or statistical channel models for UAV-assisted communication systems. For example, pure line-of-sight (LoS) channels are assumed in [7], [8], [10]-[13]. Probabilistic LoS channels are adopted in [14]-[17], where LoS and non-LoS (NLoS) conditions are probabilistically related to the elevation angle of the link between the UAV and ground UE. However, the simplified and statistical channel models are only suitable for average performance analysis, while corresponding solutions may fail to provide satisfactory performance for practical UEs in specific environments. In practice, the terrain conditions, such as buildings and other obstacles, may block air-to-ground signals and greatly deteri- 
orate the propagation environment, especially for urban areas with dense buildings [18]. In such a case, the design and optimization for UAV-assisted communication systems based on pure LoS channels or statistical channels may not be able to guarantee the performance of ground UEs. To address this problem, in [9], a UAV is first deployed to a conditional optimal position, and then the position is adjusted via a neighborhood search strategy to avoid obstacle and guarantee LoS links. In [19], an exploration-and-exploitation search strategy was developed to obtain a globally optimal UAV position over practical terrain structures for UAV relay systems with a single UE. In [20], radio map was utilized to characterize the UAV-UE channel condition. Then a jointly trajectory design, UE scheduling and bandwidth allocation optimization was performed to maximize the minimum throughput among all the UEs in a UAV-aided relay network. A geometric analysis method to detect the blockage was proposed in [21], and then a user scheduling problem was studied to decrease the blockage and enhance the spectral efficiency of a multi-UAV communication system. Although the above works take practical terrain conditions into account, there are still some limitations. The passive search in [9] may consume a long operation time. The search strategy in [19] is only appropriate for single-UE systems. The radio map required in [20], which includes the average signal strength for all combinations of UAV-UE locations, requires a large number of signal strength measurements. The geometric detection method proposed in [21] only detects blockage for a given UAV position, which does not support UAV positioning optimization.

Different from the above mentioned works, the goal of this paper is to model the blockage effect as tractable constraints using geographic information, and jointly design with UAV 3 -D positioning and resource allocation in an optimization manner, to guarantee practical communication performance in an arbitrary local area. Specifically, we consider a downlink multiuser communication system, where a UAV acts as an aerial relay to receive signals from the BS and forward them to ground UEs. Assisted by geographic information, we show that the blocked region for a BS-UAV/UAV-UE link caused by a ground building can be modeled as a polyhedron. Therefore, LoS and NLoS propagation conditions can be distinguished by evaluating whether the BS-UAV/UAV-UE link is blocked by buildings. Considering the blockage effect, an optimization problem is formulated to maximize the minimum communication capacity among ground UEs, and the corresponding solution is developed. The main contributions of this paper are summarized as follows.

1) We propose to deploy a UAV relay to improve the performance of a multiuser communication system. Assisted by geographic information, the blockage effect caused by buildings is derived to guarantee practical performance. To ensure fairness, we formulate an optimization problem to maximize the minimum communication capacity among UEs by jointly designing the positioning and the power allocation at the UAV, where the backhaul link from the ground BS to the UAV relay is also taken into consideration.
2) To solve the formulated non-convex problem, a twoloop optimization framework is developed based on Lagrangian relaxation. Specifically, the optimal Lagrangian multipliers are adaptively updated in the outer-loop to ensure the Lagrangian problem converge to the tightest upper bound on the original problem. The innerloop partitions the Lagrangian problem into a power allocation sub-problem and a UAV positioning subproblem, and optimizes them alternately by applying the block coordinate descent (BCD) technique. The optimal power allocation is obtained in closed form, while the UAV positioning sub-problem is solved approximately by utilizing the successive convex approximation (SCA) technique.

3) Simulation results show that the proposed geographic information-aided joint positioning and power allocation scheme can closely approach a performance upper bound on the considered UAV relay system and outperform two benchmark schemes.

The rest of this paper is organized as follows. In Section II, we introduce the system model, and formulate the proposed joint positioning and power allocation problem. In Section III, we transform the original problem into a more tractable Lagrangian problem. In Section IV, we propose a solution for the Lagrangian problem. The overall solution, convergence analysis, and computational complexity are discussed in Section V. Section VI presents the simulation results. Finally, the paper is concluded in Section VII.

Notation: $a$, a, $\mathbf{A}$, and $\mathcal{A}$ denote a scalar, a vector, a matrix, and a set, respectively. $\mathbb{R}^{M}$ denotes the space of $M$ dimensional real vector. $(\cdot)^{\mathrm{T}},(\cdot)^{*}$, and $(\cdot)^{\mathrm{H}}$ denote transpose, conjugate, and conjugate transpose, respectively. $\|\mathbf{a}\|$ represents the Euclidean norm of vector a. $\mathcal{A}_{2} \backslash \mathcal{A}_{1}$ represents the elements of $\mathcal{A}_{2}$ that are not included in $\mathcal{A}_{1}$. $|\mathcal{A}|$ denotes the cardinality of set $\mathcal{A}$. $\emptyset$ denotes empty set. $\overrightarrow{A B}$ denotes the vector from point $A$ to point $B \cdot \overrightarrow{A B} \cdot \overrightarrow{C D}$ and $\overrightarrow{A B} \times \overrightarrow{C D}$ denote the inner product and outer product between vector $\overrightarrow{A B}$ and vector $\overrightarrow{C D}$, respectively.

\section{System Model And Problem Formulation}

As shown in Fig. 1, we consider a downlink communication network with a single BS and $K$ ground UEs indexed by $\mathcal{K} \triangleq$ $\{1, \ldots, K\}$. To improve the communication performance of the system, one UAV is employed as a decode-and-forward (DF) relay. That is to say, the BS transmits signals to the UAV relay, and then the UAV relay forwards the signals to the ground UEs in a half-duplex mode. We assume that there are $K$ orthogonal channels for the UAV relay. As a result, there is no interference between UEs.

Without loss of generality, we employ a 3-D Cartesian coordinate system. The BS is located at $\mathbf{x}_{\mathrm{B}} \in \mathbb{R}^{3}$. It is assumed that each UE $k$ is statically located at areas without buildings and its coordinates are denoted by $\mathbf{x}_{k} \in \mathbb{R}^{3}, k \in \mathcal{K}$. The coordinates of the UAV are given by $\mathbf{x}=\left(x_{\mathrm{V}}, y_{\mathrm{V}}, h_{\mathrm{V}}\right) \in \mathbb{R}^{3}$. $M$ buildings indexed by $\mathcal{M} \triangleq\{1, \ldots, M\}$ are randomly distributed in the considered area. The BS-UAV and UAVUE links may be blocked by these buildings. As a result, the 


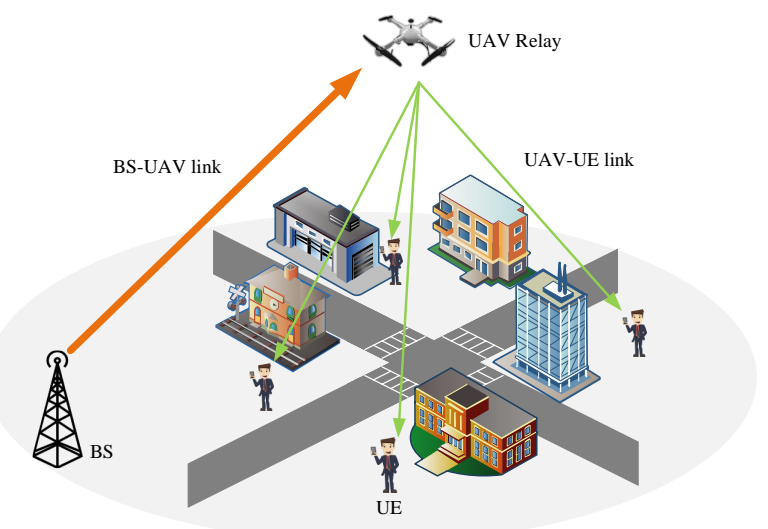

Fig. 1. Illustration of the considered UAV relay communication system.

position of the UAV relay should be carefully designed to avoid blockage. We suppose that the UAV hovers at an altitude above the tallest building in the considered region, such that no collision will occur.

\section{A. Channel Model}

Since the UAV positioning should be optimized in a relatively large timescale, we mainly focus on the large-scale channel characteristics. The distance from the BS to the UAV relay is $\left\|\mathbf{x}-\mathbf{x}_{\mathrm{B}}\right\|$, and the distance from the UAV relay to UE $k$ is $\left\|\mathbf{x}-\mathbf{x}_{k}\right\|$. Then, the channel gains of BS-UAV and UAV-UE links are respectively given by [19]

$$
\begin{aligned}
& g_{\mathrm{B}}= \begin{cases}\beta_{1}\left\|\mathbf{x}-\mathbf{x}_{\mathrm{B}}\right\|^{-\alpha_{1}}, & \operatorname{LoS}, \\
\beta_{2}\left\|\mathbf{x}-\mathbf{x}_{\mathrm{B}}\right\|^{-\alpha_{2}}, & \mathrm{NLoS},\end{cases} \\
& g_{k}= \begin{cases}\beta_{1}\left\|\mathbf{x}-\mathbf{x}_{k}\right\|^{-\alpha_{1}}, & \operatorname{LoS}, \\
\beta_{2}\left\|\mathbf{x}-\mathbf{x}_{k}\right\|^{-\alpha_{2}}, & \text { NLoS, }\end{cases}
\end{aligned}
$$

where $\alpha_{1}$ and $\alpha_{2}$ are the path loss exponents for LoS and NLoS channels, respectively. $\beta_{1}$ and $\beta_{2}$ are the channel gains at reference distance of $1 \mathrm{~m}$ for LoS and NLoS channels, respectively.

As a result, we obtain the communication capacities of the BS-UAV link and the UAV-UE link as follows:

$R_{\mathrm{B}}=W_{\mathrm{B}} \log _{2}\left(1+\frac{P_{\mathrm{B}} \beta_{i}}{N_{0} W_{\mathrm{B}}\left\|\mathbf{x}-\mathbf{x}_{\mathrm{B}}\right\|^{\alpha_{i}}}\right), i= \begin{cases}1, & \text { LoS }, \\ 2, & \mathrm{NLoS},\end{cases}$

$R_{k}=W_{\mathrm{U}} \log _{2}\left(1+\frac{P_{k} \beta_{i}}{N_{0} W_{\mathrm{U}}\left\|\mathbf{x}-\mathbf{x}_{k}\right\|^{\alpha_{i}}}\right), i= \begin{cases}1, & \text { LoS, } \\ 2, & \text { NLoS, }\end{cases}$

where $W_{\mathrm{B}}$ and $W_{\mathrm{U}}$ denote the channel bandwidths of links from the BS to the UAV and from the UAV to UE $k$, respectively. $P_{\mathrm{B}}$ denotes the transmit power of $\mathrm{BS}$ and $P_{k}$ is the transmit power of the UAV allocated to UE $k . N_{0}$ is the power spectral density of additive white Gaussian noise.

\section{B. Blockage Modeling}

To distinguish different propagation scenarios, the key is to identify whether the communication link is blocked by
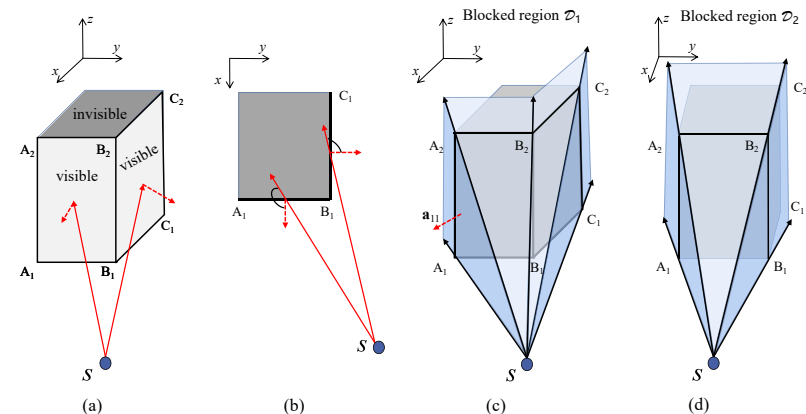

Fig. 2. Illustration of the blockage caused by building.

buildings. For $M$ buildings, $M$ and $M K$ blocked regions are generated with respect to the BS and $K$ UEs, respectively. We index these blocked regions as $\mathcal{I} \triangleq\{1, \ldots, M+M K\}$. In this paper, we assume that the shape of all the buildings is cube ${ }^{1}$. We show that giving building and UE/BS locations, the blocked region can be formulated as a polyhedron.

1) Blocked Region for the UE: As shown in Fig. 2, along the UE's line of sight, only a part of the flank surfaces of each building is visible, while other flank surfaces and the top surface are invisible to the UE. In other words, the region behind the visible flank surfaces of the building is blocked. Therefore, in order to identify the blocked region for a UE with respect to a building, the key steps are a) identifying visible flank surfaces of the building, and b) determining the boundaries of the blocked region.

According to basic geometry, the inner product between the outward normal vector of a surface and the LoS vector from a UE to any point on the surface can be utilized to judge whether the surface is visible. If the inner product is negative, the surface is visible. Otherwise, the surface is invisible [22]. A toy example is shown in Fig. 2 (a), where surface $A_{1} B_{1} B_{2} A_{2}$ and surface $B_{1} C_{1} C_{2} B_{2}$ are visible to UE $S$. The aerial view in Fig. 2 (b) gives a more visualized interpretation for the visible surfaces.

As shown in Figs. 2 (c) and (d), the boundaries of the blocked region are composed by two vertical hyperplanes and two (or one) oblique hyperplanes ${ }^{2}$, where each hyperplane is determined by the position of the UE and two adjacent vertices on the flank surface of the building. Since a hyperplane determines two halfspaces, by applying analytic geometry theory, the blocked region can be represented by the intersection of a finite number of halfspaces, which is polyhedron [23]. The $i$-th blocked region is

$$
\mathcal{D}_{i}=\left\{\mathbf{x} \in \mathbb{R}^{3} \mid \mathbf{a}_{i j}^{\mathrm{T}} \mathbf{x}-b_{i j} \leq 0, \forall j \in \mathcal{J}_{i}\right\}
$$

where $\mathcal{J}_{i}$ is the set of indexes of hyperplanes for the $i$-th blocked region. $\left|\mathcal{J}_{i}\right|$ equals to four or three, corresponding to situations in Figs. 2 (c) and (d). $\mathbf{a}_{i j} \in \mathbb{R}^{3}$ is the outward normal vector of hyperplane $j$, which can be obtained by the outer product of any two non-collinear vectors in the

\footnotetext{
${ }^{1}$ For other types of building, we can always find a cube envelope which covers the building, and thus the proposed solution is workable.

${ }^{2}$ The number of oblique hyperplanes equals to the number of visible flank surfaces.
} 
plane, pointing to the outside of the blocked region. Constant $b_{i j} \in \mathbb{R}$ determines the offset of hyperplane $j$ from the origin point, which can be obtained by the inner product between $\mathbf{a}_{i j}$ and any point in the hyperplane. For example, in Fig. 2 (c), the outer boundaries of the blocked region $\mathcal{D}_{1}$ are hyperplane $S A_{1} A_{2}, S A_{2} B_{2}, S B_{2} C_{2}$, and $S C_{2} C_{1}$. The outward normal vector $\mathbf{a}_{11}=\frac{\overrightarrow{S A_{2}} \times \overrightarrow{S A_{1}}}{\left\|\overrightarrow{S A_{2}} \times \overrightarrow{S A_{1}}\right\|}$, and the corresponding offset $b_{11}=\mathbf{a}_{11}^{\mathrm{T}} \cdot \overrightarrow{O S}$, where $O$ denotes the origin point.

In summary, given the location of the UE and the vertices of a building, the algorithm for obtaining the blocked region is shown in Algorithm 1.

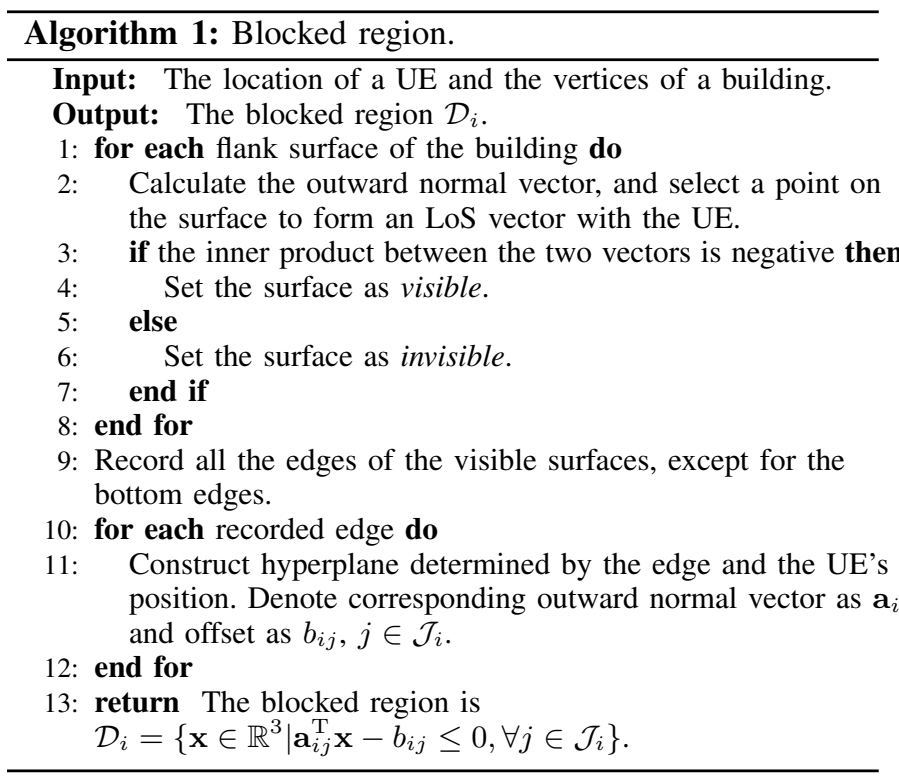

2) Blocked Region for the BS: Depending on the relative height of the BS and the buildings, there are two possible situations for the blocked region. If the BS antenna is deployed higher than the buildings, no blockage exists between the BS and the UAV relay. The blocked region is an empty set, i.e., $\mathcal{D}_{i}=\emptyset$. On the other hand, if the BS antenna is lower than the buildings, the blockage modeling method for the UE in Algorithm 1 can be directly used for the BS.

\section{Problem Formulation}

To ensure the fairness, we aim to maximize the minimum communication capacity among all the UEs by jointly optimizing the UAV positioning and the power allocation, subject to the blockage effect of buildings, and the backhaul constraint. Since the NLoS environment leads to dramatic deterioration of the communication capacity, it is preferred to avoid all blocked regions to guarantee the overall performance. The problem is formulated as

$$
\begin{aligned}
\max _{\mathbf{x}, P_{\mathrm{B}},\left\{P_{k}\right\}} & R \\
\text { s.t. } \quad & R \leq R_{k}, \forall k \in \mathcal{K}, \\
& K R \leq R_{\mathrm{B}}, \\
& P_{k} \geq 0, \forall k \in \mathcal{K}, \\
& \sum_{k \in \mathcal{K}} P_{k} \leq P_{\mathrm{V}}^{\text {tot }}, \\
& 0 \leq P_{\mathrm{B}} \leq P_{\mathrm{B}}^{\text {tot }}, \\
& \mathbf{x} \in \mathcal{D} \backslash \mathcal{D}_{i}, \forall i \in \mathcal{I},
\end{aligned}
$$

where $R$ denotes the minimum communication capacity among all the UEs. $\mathcal{D}=\left\{\mathbf{x}=\left(x_{\mathrm{V}}, y_{\mathrm{V}}, h_{\mathrm{V}}\right) \mid x_{\mathrm{V}} \in\right.$ $\left.\left[0, x_{\mathrm{D}}\right], y_{\mathrm{V}} \in\left[0, y_{\mathrm{D}}\right], h_{\mathrm{V}} \geq h_{\min }\right\}$ denotes the whole considered region, and $\mathcal{D}_{i}$ is the $i$-th blocked region for $i \in \mathcal{I}$. Constraint (6a) indicates that the minimum communication capacity does not exceed the communication capacity of each UAV-UE link. Constraint (6b) ensures that the BSUAV backhaul link is capable to support all UEs with the minimum communication capacity. Constraints (6c), (6d), and (6e) indicate that the transmit powers are nonnegative and do not exceed a maximum value, where $P_{\mathrm{B}}^{\text {tot }}$ and $P_{\mathrm{V}}^{\text {tot }}$ are the maximum transmit powers of the BS and the UAV relay, respectively. Constraint (6f) confines that the UAV has to be deployed to avoid the blocked regions for the BS-UAV and UAV-UE links. In other words, under constraint (6f), the LoS propagation environment is always guaranteed for the BS-UAV and UAV-UE links ${ }^{3}$.

\section{PRoblem TRANSFORMATION}

Due to the non-convex and intractable constraints, the original problem (6) is challenging to solve. In this section, we first transform the blockage constraint into tractable mixed-integer linear constraints. Then, Lagrangian relaxation is introduced to relax the binary variables to continuous variables thus leading to a Lagrangian problem.

\section{A. Transformation of the Blockage Constraint}

To make constraint (6f) more tractable, we introduce auxiliary binary variable $l_{i j}$ and provide the following theorem.

Theorem 1. For each $i \in \mathcal{I}, \mathbf{x} \in \mathcal{D} \backslash \mathcal{D}_{i}$ is equivalent to

$$
\left\{\begin{array}{l}
\mathbf{a}_{i j}^{T} \mathbf{x}-b_{i j}+C l_{i j}>0, \forall j \in \mathcal{J}_{i}, \\
l_{i j} \in\{0,1\}, \forall j \in \mathcal{J}_{i}, \\
\sum_{j \in \mathcal{J}_{i}} l_{i j} \leq\left|\mathcal{J}_{i}\right|-1, \\
\mathbf{x} \in \mathcal{D},
\end{array}\right.
$$

where $C$ is a sufficiently large constant.

Proof. On one hand, we verify that any point $\mathbf{x} \in \mathcal{D} \backslash \mathcal{D}_{i}$ satisfies constraint (7). The definition of $\mathcal{D} \backslash \mathcal{D}_{i}$ is given by $\mathcal{D} \backslash \mathcal{D}_{i}=\left\{\mathbf{x} \in \mathcal{D} \mid \mathbf{a}_{i j}^{T} \mathbf{x}-b_{i j}>0, \exists j \in \mathcal{J}_{i}\right\}$. With involving

\footnotetext{
${ }^{3}$ We assume that all UEs are located at areas without buildings. Therefore, the feasible region for UAV positioning is nonempty as there always exists an unblocked region for sufficient high altitude of the UAV relay.
} 
auxiliary binary variable $l_{i j} \in\{0,1\}, \forall j \in \mathcal{J}_{i}$, we know that $\exists j \in \mathcal{J}_{i}$ such that $\mathbf{a}_{i j}^{T} \mathbf{x}-b_{i j}+C l_{i j}>0$ by setting $l_{i j}=0$. For other $j \in \mathcal{J}_{i}, \mathbf{a}_{i j}^{T} \mathbf{x}-b_{i j}+C l_{i j}>0$ holds by setting $l_{i j}=1$, for a sufficiently large $C>0$. Thus, $\forall j \in \mathcal{J}_{i}, \mathbf{a}_{i j}^{T} \mathbf{x}-b_{i j}+C l_{i j}>0$ holds, and there exists at least one $l_{i j}=0$, which makes $\sum_{j \in \mathcal{J}_{i}} l_{i j} \leq\left|\mathcal{J}_{i}\right|-1$ hold.

On the other hand, $l_{i j} \in\{0,1\}, \forall j \in \mathcal{J}_{i}$ and $\sum_{j \in \mathcal{J}_{i}} l_{i j} \leq$ $\left|\mathcal{J}_{i}\right|-1$ constrains that $\exists j \in \mathcal{J}_{i}, l_{i j}=0$. Together with $\mathbf{x} \in$ $\mathcal{D}$ and $\mathbf{a}_{i j}^{T} \mathbf{x}-b_{i j}+C l_{i j}>0, \forall j \in \mathcal{J}_{i}$, the position $\mathbf{x}$ is constrained in a region where $\mathbf{x} \in \mathcal{D}$ and $\exists j \in \mathcal{J}_{i}, \mathbf{a}_{i j}^{T} \mathbf{x}-$ $b_{i j}+C l_{i j}=\mathbf{a}_{i j}^{T} \mathbf{x}-b_{i j}+0>0$, which is the definition of $\mathcal{D} \backslash \mathcal{D}_{i}$.

This completes the proof.

According to Theorem 1, constraint (6f) is equivalent to the following mixed integer linear constraints:

$$
\begin{aligned}
& \mathbf{a}_{i j}^{T} \mathbf{x}-b_{i j}+C l_{i j}>0, \forall j \in \mathcal{J}_{i}, \forall i \in \mathcal{I}, \\
& l_{i j} \in\{0,1\}, \forall j \in \mathcal{J}_{i}, \forall i \in \mathcal{I}, \\
& \sum_{j \in \mathcal{J}_{i}} l_{i j} \leq\left|\mathcal{J}_{i}\right|-1, \forall i \in \mathcal{I}, \\
& \mathbf{x} \in \mathcal{D},
\end{aligned}
$$

where $C$ is a sufficiently large constant.

\section{B. Lagrangian Relaxation}

By replacing constraint (6f) with constraints (8)-(11), problem (6) is equivalent to

$$
\begin{aligned}
\max _{\mathbf{x}, P_{\mathrm{B}},\left\{P_{k}\right\},\left\{l_{i j}\right\}} & R \\
\text { s.t. } & (6 \mathrm{a}),(6 \mathrm{~b}),(6 \mathrm{c}),(6 \mathrm{~d}),(6 \mathrm{e}), \\
& (8),(9),(10),(11) .
\end{aligned}
$$

Due to the non-convexity of constraints (6a), (6b) and binary constraint (9), problem (12) is a non-convex and mixed-integer problem, which is difficult to obtain a globally optimal solution. Therefore, we propose to utilize Lagrangian relaxation [24]. First, binary constraint (9) can be equivalently replaced by

$$
\begin{gathered}
0 \leq l_{i j} \leq 1, \forall j \in \mathcal{J}_{i}, \forall i \in \mathcal{I}, \\
\sum_{j \in \mathcal{J}_{i}} l_{i j}\left(1-l_{i j}\right) \leq 0, \forall i \in \mathcal{I} .
\end{gathered}
$$

In this way, $l_{i j}$ becomes a continuous optimization variable between 0 and 1 . Furthermore, by introducing Lagrangian multipliers $\lambda_{i}$ for $i \in \mathcal{I}$, we obtain a Lagrangian problem

$$
\begin{aligned}
\max _{\mathbf{x}, P_{\mathrm{B}},\left\{P_{k}\right\},\left\{l_{i j}\right\}} & R-\sum_{i \in \mathcal{I}} \lambda_{i} \sum_{j \in \mathcal{J}_{i}} l_{i j}\left(1-l_{i j}\right) \\
\text { s.t. } \quad & \lambda_{i} \geq 0, \forall i \in \mathcal{I}, \\
& (6 \mathrm{a}),(6 \mathrm{~b}),(6 \mathrm{c}),(6 \mathrm{~d}),(6 \mathrm{e}), \\
& (8),(10),(11),(13) .
\end{aligned}
$$

For any given Lagrangian multipliers, the optimal solution for Lagrangian problem (15) is an upper bound on original problem (12) [24]. To decrease the duality gap and obtain a feasible solution to original problem (12), the Lagrangian

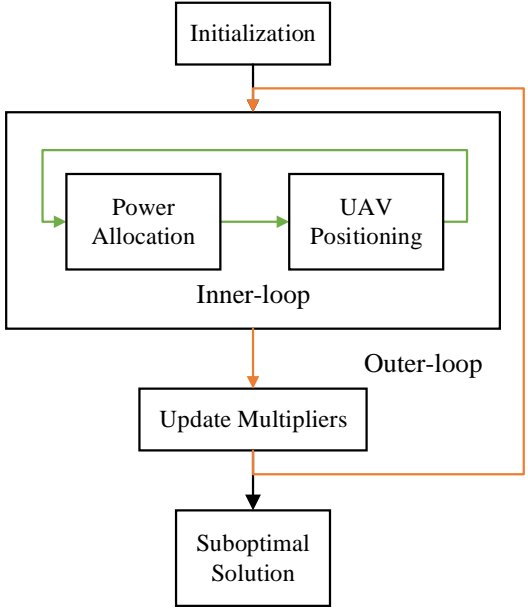

Fig. 3. The flowchart of two-loop solution for Lagrangian problem (15).

multipliers need to be adjusted. Next, we develop a two-loop optimization framework to adaptively determine the values of Lagrangian multipliers as well as the UAV positioning and power allocation.

\section{SOLUTION OF THE LAGRANGIAN PROBLEM}

In this section, we introduce the method to solve Lagrangian problem (15) as well as adjusting the Lagrangian multipliers to deduce a good feasible solution to the original problem (12). A two-loop optimization framework is developed, where the inner-loop optimizes the Lagrangian problem (15) for given Lagrangian multipliers, and the outer-loop updates the multipliers to decrease the duality gap. The flowchart of the solution is shown in Fig. 3. Specifically, due to the nonconcave objective function and non-convex constraints in (6a) and (6b), Lagrangian problem (15) is not a convex problem and is challenging to obtain a globally optimal solution. Therefore, we develop an iterative algorithm by applying the BCD technique [25], [26]. For given UAV position $\mathrm{x}$ and the auxiliary variables $\left\{l_{i j}\right\}$, we solve the power allocation subproblem and obtain a closed-form solution. For given transmit powers $\left\{P_{k}\right\}$ and $P_{\mathrm{B}}$, we solve the UAV positioning subproblem approximately by utilizing the SCA technique [27]. These two sub-problems are alternately optimized until a locally optimal solution is obtained, which are discussed in Sections IV-A and IV-B. The method to update Lagrangian multipliers is discussed in Section IV-C.

\section{A. Power Allocation}

For the $T$-th outer-loop, given Lagrangian multipliers $\left\{\lambda_{i}^{(T)}\right\}$, we develop an iterative algorithm to solve Lagrangian problem (15). For the $t$-th iteration of the inner-loop, given UAV's position $\mathbf{x}^{(t)}$ and auxiliary variables $\left\{l_{i j}^{(t)}\right\}$, prob- 
lem (15) is transformed to the following power allocation problem

$$
\begin{aligned}
\max _{P_{\mathrm{B}},\left\{P_{k}\right\}} & R-\sum_{i \in \mathcal{I}} \lambda_{i}^{(T)} \sum_{j \in \mathcal{J}_{i}} l_{i j}^{(t)}\left(1-l_{i j}^{(t)}\right) \\
\text { s.t. } \quad & R \leq W_{\mathrm{U}} \log _{2}\left(1+\eta_{k}^{(t)} P_{k}\right), \forall k \in \mathcal{K}, \\
& K R \leq W_{\mathrm{B}} \log _{2}\left(1+\eta_{\mathrm{B}}^{(t)} P_{\mathrm{B}}\right) \\
& (6 \mathrm{c}),(6 \mathrm{~d}),(6 \mathrm{e})
\end{aligned}
$$

where $\eta_{k}^{(t)}=\beta_{1} /\left(N_{0} W_{\mathrm{U}}\left\|\mathbf{x}^{(t)}-\mathbf{x}_{k}\right\|^{\alpha_{1}}\right)$ and $\eta_{\mathrm{B}}^{(t)}=$ $\beta_{1} /\left(N_{0} W_{\mathrm{B}}\left\|\mathbf{x}^{(t)}-\mathbf{x}_{\mathrm{B}}\right\|^{\alpha_{1}}\right)$ are intermediate parameters for notational simplicity. The second term in the objective function is a constant which does not impact the optimality. According to constraint (6f) and its equivalent formulas, the UAV is restricted in a region without any building blockage, to establish LoS channels for the BS-UAV and UAV-UE links. Therefore, parameters $\alpha_{1}$ and $\beta_{1}$ are adopted for solving problem (16).

Problem (16) is a convex problem with respect to $P_{\mathrm{B}}$ and $\left\{P_{k}\right\}$ [28], [29]. To maximize the minimum communication capacity with minimal transmit powers, we provide the following theorem.

Theorem 2. For given position $\mathbf{x}^{(t)}$ and auxiliary variables $\left\{l_{i j}^{(t)}\right\}$, the optimal power allocation for the BS and UAV relay is given as follows:

$$
\begin{aligned}
& \text { If }\left(1+\eta_{\mathrm{B}}^{(\mathrm{t})} \mathrm{P}_{\mathrm{B}}^{\mathrm{tot}}\right)^{\frac{\mathrm{W}_{\mathrm{B}}}{\mathrm{K}}}<\left(1+\eta_{\mathrm{V}}^{(\mathrm{t})} \mathrm{P}_{\mathrm{V}}^{\mathrm{tot}}\right)^{\mathrm{W}_{\mathrm{U}}}, \\
& \left\{\begin{array}{l}
P_{\mathrm{B}}^{(t+1)}=P_{\mathrm{B}}^{\mathrm{tot}} \\
P_{k}^{(t+1)}=\left(\left(1+\eta_{\mathrm{B}}^{(t)} P_{\mathrm{B}}^{\mathrm{tot}}\right)^{\frac{W_{\mathrm{B}}}{K W_{\mathrm{U}}}}-1\right) / \eta_{k}^{(t)} ;
\end{array}\right.
\end{aligned}
$$

otherwise,

$$
\begin{aligned}
& \left\{\begin{array}{l}
P_{\mathrm{B}}^{(t+1)}=\left(\left(1+\eta_{\mathrm{V}}^{(t)} P_{\mathrm{V}}^{\mathrm{tot}}\right)^{\frac{K W_{\mathrm{U}}}{W_{\mathrm{B}}}}-1\right) / \eta_{\mathrm{B}}^{(t)}, \\
P_{k}^{(t+1)}=\eta_{\mathrm{V}}^{(t)} P_{\mathrm{V}}^{\mathrm{tot}} / \eta_{k}^{(t)},
\end{array}\right. \\
& \text { with } \eta_{\mathrm{V}}^{(t)}=1 / \sum_{k \in \mathcal{K}} \frac{1}{\eta_{k}^{(t)}} .
\end{aligned}
$$

Proof. See Appendix A.

Hereto, we have obtained the optimal solution for the power allocation sub-problem.

\section{B. UAV Positioning}

Given the power allocation $\left\{P_{k}^{(t+1)}\right\}$ and $P_{\mathrm{B}}^{(t+1)}$, problem (15) is transformed to the following positioning problem

$$
\begin{aligned}
\max _{\mathbf{x},\left\{l_{i j}\right\}} & R-\sum_{i \in \mathcal{I}} \lambda_{i}^{(T)} \sum_{j \in \mathcal{J}_{i}} l_{i j}\left(1-l_{i j}\right) \\
\text { s.t. } & R \leq W_{\mathrm{U}} \log _{2}\left(1+\zeta_{k}^{(t)} /\left\|\mathbf{x}-\mathbf{x}_{k}\right\|^{\alpha_{1}}\right), \forall k \in \mathcal{K}, \\
& K R \leq W_{\mathrm{B}} \log _{2}\left(1+\zeta_{\mathrm{B}}^{(t)} /\left\|\mathbf{x}-\mathbf{x}_{\mathrm{B}}\right\|^{\alpha_{1}}\right)
\end{aligned}
$$

(8), (10), (11), (13), where $\zeta_{k}^{(t)}=\frac{P_{k}^{(t+1)} \beta_{1}}{N_{0} W_{\mathrm{U}}}$ and $\zeta_{\mathrm{B}}^{(t)}=\frac{P_{\mathrm{B}}^{(t+1)} \beta_{1}}{N_{0} W_{\mathrm{B}}}$ are intermediate parameters for notational simplicity. Problem (18) is not a concave/convex problem because of the non-concave objective function and the non-convex constraints in (18a) and (18b). In the following, we employ the SCA technique to obtain a suboptimal solution.

Considering the objective function, for a given local point $l_{i j}^{(t)}$ in the $t$-th iteration, we have the following lower bound on $\left(l_{i j}\right)^{2}$ :

$$
\left(l_{i j}\right)^{2} \geq 2 l_{i j}^{(t)} l_{i j}-\left(l_{i j}^{(t)}\right)^{2} .
$$

Considering constraints (18a) and (18b), note that function $r(z)=\log _{2}\left(1+1 / z^{\alpha_{1}}\right)$ is convex with respect to $z$ for $z>0$, thus the right-hand-side (RHS) of constraint (18a) and the RHS of constraint (18b) are convex with respect to $\left\|\mathbf{x}-\mathbf{x}_{k}\right\|$ and $\left\|\mathbf{x}-\mathbf{x}_{\mathrm{B}}\right\|$, respectively. For a given local point $\mathbf{x}^{(t)}$ in the $t$-th iteration, the RHS of constraint (18a) is lower-bounded by its first-order Taylor expansion at $\left\|\mathbf{x}^{(t)}-\mathbf{x}_{k}\right\|$ as

$$
\begin{aligned}
& W_{\mathrm{U}} \log _{2}\left(1+\zeta_{k}^{(t)} /\left\|\mathbf{x}-\mathbf{x}_{k}\right\|^{\alpha_{1}}\right) \geq \\
& A_{k}^{(t)}-B_{k}^{(t)}\left(\left\|\mathbf{x}-\mathbf{x}_{k}\right\|-\left\|\mathbf{x}^{(t)}-\mathbf{x}_{k}\right\|\right) \triangleq R_{k}^{\mathrm{lb}},
\end{aligned}
$$

with

$$
\begin{aligned}
& A_{k}^{(t)}=W_{\mathrm{U}} \log _{2}\left(1+\zeta_{k}^{(t)} /\left\|\mathbf{x}^{(t)}-\mathbf{x}_{k}\right\|^{\alpha_{1}}\right), \\
& B_{k}^{(t)}=\frac{W_{\mathrm{U}} \zeta_{k}^{(t)} \alpha_{1}}{\left\|\mathbf{x}^{(t)}-\mathbf{x}_{k}\right\|\left(\left\|\mathbf{x}^{(t)}-\mathbf{x}_{k}\right\|^{\alpha_{1}}+\zeta_{k}^{(t)}\right) \ln 2} .
\end{aligned}
$$

The RHS of constraint (18b) is lower-bounded by its first-order Taylor expansion at $\left\|\mathbf{x}^{(t)}-\mathbf{x}_{\mathrm{B}}\right\|$ as

$$
\begin{aligned}
& W_{\mathrm{B}} \log _{2}\left(1+\zeta_{\mathrm{B}}^{(t)} /\left\|\mathbf{x}-\mathbf{x}_{\mathrm{B}}\right\|^{\alpha_{1}}\right) \geq \\
& A_{\mathrm{B}}^{(t)}-B_{\mathrm{B}}^{(t)}\left(\left\|\mathbf{x}-\mathbf{x}_{\mathrm{B}}\right\|-\left\|\mathbf{x}^{(t)}-\mathbf{x}_{\mathrm{B}}\right\|\right) \triangleq R_{\mathrm{B}}^{\mathrm{lb}},
\end{aligned}
$$

with

$$
\begin{aligned}
& A_{\mathrm{B}}^{(t)}=W_{\mathrm{B}} \log _{2}\left(1+\zeta_{\mathrm{B}}^{(t)} /\left\|\mathbf{x}^{(t)}-\mathbf{x}_{\mathrm{B}}\right\|^{\alpha_{1}}\right), \\
& B_{\mathrm{B}}^{(t)}=\frac{W_{\mathrm{B}} \zeta_{\mathrm{B}}^{(t)} \alpha_{1}}{\left\|\mathbf{x}^{(t)}-\mathbf{x}_{\mathrm{B}}\right\|\left(\left\|\mathbf{x}^{(t)}-\mathbf{x}_{\mathrm{B}}\right\|^{\alpha_{1}}+\zeta_{\mathrm{B}}^{(t)}\right) \ln 2} .
\end{aligned}
$$

As a result, given local points $\mathbf{x}^{(t)}$ and $\left\{l_{i j}^{(t)}\right\}$ in the $t$-th iteration, problem (18) is relaxed as

$$
\begin{aligned}
\max _{\mathbf{x},\left\{l_{i j}\right\}} & R-\sum_{i \in \mathcal{I}} \lambda_{i}^{(T)} \sum_{j \in \mathcal{J}_{i}}\left(l_{i j}-2 l_{i j}^{(t)} l_{i j}+\left(l_{i j}^{(t)}\right)^{2}\right) \\
\text { s.t. } \quad & R \leq R_{k}^{\mathrm{lb}}, \forall k \in \mathcal{K}, \\
& K R \leq R_{\mathrm{B}}^{\mathrm{lb}}, \\
& \left\|\mathbf{x}-\mathbf{x}^{(t)}\right\| \leq \rho^{(t)},
\end{aligned}
$$$$
\text { (8), (10), (11), (13). }
$$

It is easy to verify that problem (26) is a convex problem and can be readily solved by standard convex program solvers, such as CVXPY [29], [30]. The objective function of problem (26) is a lower bound on the objective function of problem (18). Constraints (26a) and (26b) have tighter forms compared to constraints (18a) and (18b). Hance, any feasible solution of problem (26) is also feasible for problem (18). Constraint (26c) is introduced to limit the update of $\mathbf{x}^{(t+1)}$ in 
a small neighborhood around the local point $\mathbf{x}^{(t)} \cdot \rho^{(t)}$ is the radius of the spherical region and is gradually reduced during the iteration to ensure convergence. A feasible way to update $\rho^{(t)}$ is $\rho^{(t+1)}=\kappa_{1} \rho^{(t)}$, where $\kappa_{1}<1$ is the stepsize.

\section{Updating Lagrangian Multipliers}

To decrease the duality gap between Lagrangian problem (15) and original problem (12), the Lagrangian multipliers need to be adjusted. This is actually a dual problem with respect to Lagrangian multipliers, which can be optimized by the gradient method [31]. For the $T$-th outer-loop, we have gotten the converged solution to Lagrangian problem (15) for given $\left\{\lambda_{i}^{(T)}\right\}$ by inner-loop iterations, denoted by $\overline{\mathbf{x}}^{(T)},\left\{\bar{l}_{i j}^{(T)}\right\}$, $\bar{P}_{\mathrm{B}}^{(T)}$ and $\left\{\bar{P}_{i}^{(T)}\right\}$. Then the multiplier $\lambda_{i}$ is updated by using the following formula [24]:

$$
\lambda_{i}^{(T+1)}=\max \left(0, \lambda_{i}^{(T)}+\gamma^{(T)} \sum_{j \in \mathcal{J}_{i}} \bar{l}_{i j}^{(T)}\left(1-\bar{l}_{i j}^{(T)}\right)\right),
$$

where $\gamma^{(T)}$ is the stepsize formulated as

$$
\gamma^{(T)}=\frac{2 \times\left(q_{\mathrm{U}}^{(T)}-q_{\mathrm{L}}^{(T)}\right)}{\sum_{i \in \mathcal{I}}\left(\sum_{j \in \mathcal{J}_{i}} \bar{l}_{i j}^{(T)}\left(1-\bar{l}_{i j}^{(T)}\right)\right)^{2}},
$$

where $q_{\mathrm{U}}^{(T)}$ is the objective value of Lagrangian problem (15) at the $T$-th outer-loop, $q_{\mathrm{L}}^{(T)}$ is the objective value of original problem (12). The denominator is the sum square of the gradients. If the solution to Lagrangian problem (15) is not feasible for original problem (12), which means the BS-UAV link or UAV-UE link is blocked, $q_{\mathrm{L}}^{(T)}$ is estimated to be zero. Otherwise, $q_{\mathrm{L}}^{(T)}$ is set to be the corresponding communication capacity by substituting the solution into original problem (12).

\section{Overall Solution}

In this section, we first provide the overall solution of the joint positioning and power allocation problem for UAV relay systems aided by geographic information. Then, the convergence and computational complexity are analyzed.

\section{A. Overall Solution}

The overall solution of the algorithm is summarized in Algorithm 2. In Line 1, we invoke Algorithm 1 to calculate the blocked regions for the BS and $K$ UEs caused by $M$ buildings based on geographic information. Then, in Lines 3-14, we employ the Lagrangian relaxation framework and update the Lagrangian multipliers $\left\{\lambda_{i}\right\}$ in an iterative way. Lines 5-10 solve Lagrangian problem (15) with given $\left\{\lambda_{i}^{(T)}\right\}$, where the power allocation and UAV positioning are optimized in an alternate manner. The inner-loop terminates, when the increase of the objective value of problem (26) from one iteration to the next falls bellow a threshold $\epsilon_{\mathrm{t}}$ or the number of iterations exceeds the maximum value $L_{\mathrm{t} \text {,max }}$. The outer-loop terminates, when the difference between the objective value of problem (15) $q_{\mathrm{U}}^{(T)}$ and the objective value of problem (12) $q_{\mathrm{L}}^{(T)}$ falls bellow a threshold $\epsilon_{\mathrm{T}}$ or the number of iterations exceeds the maximum value $L_{\mathrm{T}, \max }$.

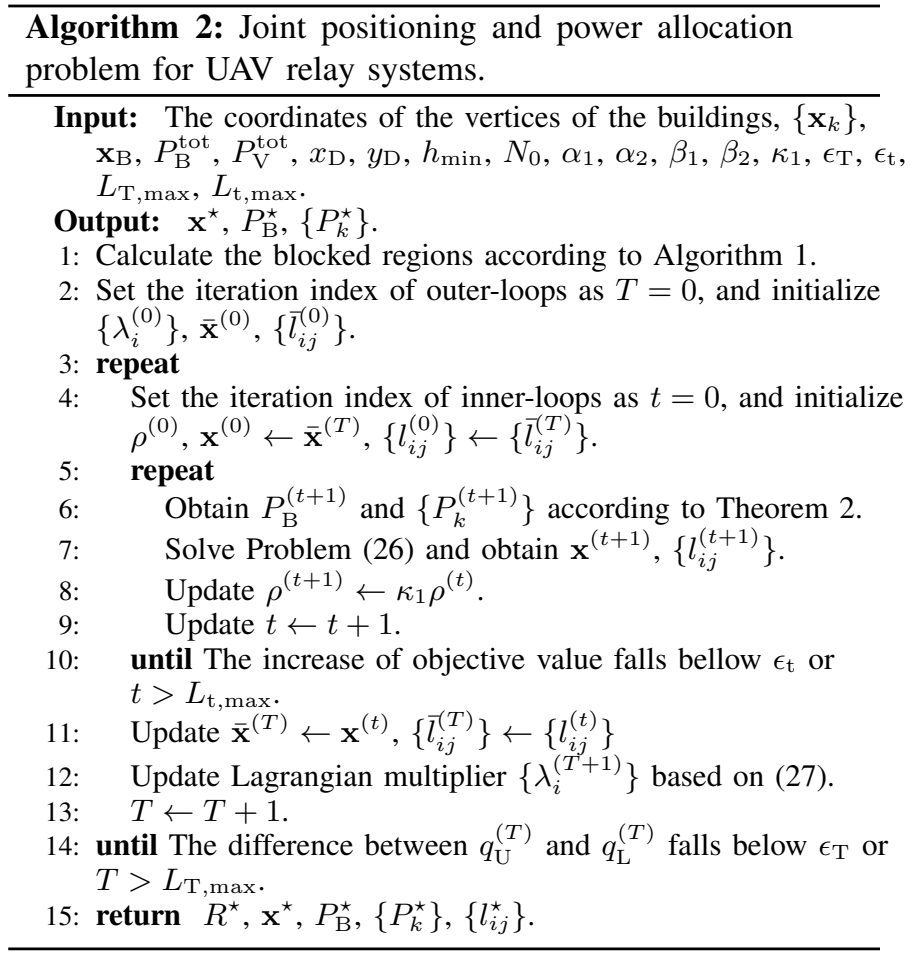

\section{B. Convergence Analysis}

1) Inner-loop Convergence: As discussed in Section IV, Lagrangian problem (15) is solved iteratively by applying BCD and SCA techniques. We first show that the objective function value of Lagrangian problem (15) converges to a finite value. We use $q_{\text {pos }}^{\mathrm{lb}}$ to represent the objective values of approximate positioning problem (26). Denote $\mathbf{P}=\left\{P_{\mathrm{B}},\left\{P_{k}, \forall k \in\right.\right.$ $\mathcal{K}\}\}, \mathbf{Q}=\left\{\mathbf{x},\left\{l_{i j}, \forall i \in \mathcal{I}, \forall j \in \mathcal{J}_{i}\right\}\right\}$. On one hand, in Line 6 of Algorithm 2, since the closed-form optimal solution of (16) is obtained for given $\mathbf{Q}^{(t)}$, we have

$$
q_{\mathrm{U}}\left(\mathbf{P}^{(t)}, \mathbf{Q}^{(t)}\right) \leq q_{\mathrm{U}}\left(\mathbf{P}^{(t+1)}, \mathbf{Q}^{(t)}\right)
$$

On the other hand, for given $\mathbf{P}^{(t+1)}$ in Line 7 of Algorithm 2, we have

$$
\begin{aligned}
q_{\mathrm{U}}\left(\mathbf{P}^{(t+1)}, \mathbf{Q}^{(t)}\right) & \stackrel{(\mathrm{a})}{=} q_{\mathrm{pos}}^{\mathrm{lb}}\left(\mathbf{P}^{(t+1)}, \mathbf{Q}^{(t)}\right) \\
& \stackrel{\text { (b) }}{\leq} q_{\mathrm{pos}}^{\mathrm{lb}}\left(\mathbf{P}^{(t+1)}, \mathbf{Q}^{(t+1)}\right) \\
& \stackrel{\text { (c) }}{\leq} q_{\mathrm{U}}\left(\mathbf{P}^{(t+1)}, \mathbf{Q}^{(t+1)}\right)
\end{aligned}
$$

where (a) holds because the first-order Taylor expansions in (19), (20) and (23) are tight at $\mathbf{Q}^{(t)}$. (b) holds because $\mathbf{Q}^{(t+1)}$ is the optimal solution of problem (26) for given $\mathbf{P}^{(t+1)}$ and $\mathbf{Q}^{(t)}$. (c) holds because the objective value of problem (26) is the lower bound on that of its original problem (18) at $\mathbf{Q}^{(t+1)}$, under small neighborhood constraint (26c). Based on (29) and (30), we have

$$
q_{\mathrm{U}}\left(\mathbf{P}^{(t)}, \mathbf{Q}^{(t)}\right) \leq q_{\mathrm{U}}\left(\mathbf{P}^{(t+1)}, \mathbf{Q}^{(t+1)}\right)
$$

which indicates that the objective value of problem (15) is non-decreasing during the iteration. Since the objective value is upper bounded by a finite value, it finally converges to a finite value. 
Besides, under small neighborhood constraint (26c), the update of $\mathbf{x}^{(t+1)}$ is restricted in a small neighborhood around the local point $\mathbf{x}^{(t)}$ with a decreasing radius $\rho^{(t)}$. Therefore, we have

$$
\lim _{t \rightarrow \infty}\left\|\mathbf{x}^{(t+1)}-\mathbf{x}^{(t)}\right\| \leq \lim _{t \rightarrow \infty} \rho^{(t)}=0 .
$$

Consequently, the solution of Lagrangian problem (15) converges to a point.

2) Outer-loop Convergence: Algorithm 2 is designed to solve a dual problem with respect to the Lagrangian multipliers. The dual value is guaranteed to be decreased during iterations by using the gradient method [31], and it is lower bounded by zero. Therefore, the outer-loop iteration is guaranteed to converge.

\section{Computational Complexity}

In the proposed joint UAV positioning and power allocation algorithm, Line 1 in Algorithm 2 needs to calculate $M+K M$ blocked regions, which entails a computational complexity of $\mathcal{O}(K M)$. The main computational complexity of Lines 5-10 comes from solving positioning sub-problem (26), whose complexity is $\mathcal{O}\left((K M)^{3.5}\right)$ by using the interior point method [23]. Line 12 needs to estimate whether the solution of Lagrangian problem (15) is feasible for the original problem (6), i.e., whether the UAV's position is out of all the $M+K M$ blocked regions, which entails a computational complexity of $\mathcal{O}(K M)$. As a result, the overall computational complexity of Algorithm 2 is $\mathcal{O}\left(L_{\mathrm{T}, \max } L_{\mathrm{t}, \max }(K M)^{3.5}\right)$.

\section{PERformance EVAluation}

In this section, we provide simulation results to evaluate the performance of the proposed joint UAV positioning and power allocation scheme for UAV relay systems aided by geographic information.

\section{A. Simulation Setup and Benchmark Schemes}

We consider a Manhattan-like dense urban area with size $500 \times 500 \mathrm{~m}^{2}$, i.e., $x_{\mathrm{D}}=500, y_{\mathrm{D}}=500$. The center of each building follows $\left(100 K_{x}-50,100 K_{y}-50\right)$, with $K_{x}=1, \ldots, 5, K_{y}=1, \ldots, 5$. The length and width of each building are random variables which follow a uniform distribution in a range which is properly determined to reach the desired $20 \%$ building density, i.e., the ratio of builtup land area to the total land area [32]. The heights of the buildings are random variables that follow a Rayleigh distribution with an average height of 23 meters, where the distribution function is interceptive between 3 meters to 50 meters. Thus, the minimum flight altitude of the UAV relay $h_{\text {min }}$ is set to 50 meters. The coordinates of the BS antenna are set as $\mathbf{x}_{\mathrm{B}}=(0,0,25)$. We adopt the channel models in (1) and (2), where an LoS path exists for the BS-UAV and UAVUE links if the UAV is located out of the blocked regions. Otherwise, NLoS channels are employed. In (8), a sufficiently large constant $C$ is set to

$$
C=\max _{\forall i \in \mathcal{I}, \forall j \in \mathcal{J}_{i}} 5\left(b_{i j}-\mathbf{a}_{i j}^{T} \mathbf{x}\right), \text { s.t. } \mathbf{x} \in \mathcal{D} .
$$

Auxiliary variable $l_{i j}$ is initialized as $l_{i j}^{(0)}=\left(\left|\mathcal{J}_{i}\right|-\right.$ $1) /\left|\mathcal{J}_{i}\right|, \forall j \in \mathcal{J}_{i}, \forall i \in \mathcal{I}$. The position of UAV relay is initialized as $\mathbf{x}^{(0)}=\left(\frac{x_{\mathrm{D}}}{2}, \frac{y_{\mathrm{D}}}{2}, 300\right)$. In this way, the initial values are a feasible solution to problem (26). Other adopted simulation parameter settings are summarized in Table I [32], [33], unless specified otherwise. The UEs are randomly generated in the areas without buildings, and each point in the simulation figures is the average performance over $500 \mathrm{UE}$ distributions.

TABLE I

Simulation PARAMETERS

\begin{tabular}{|c|l|c|}
\hline Parameter & \multicolumn{1}{|c|}{ Description } & Value \\
\hline$P_{\mathrm{B}}^{\mathrm{tot}}$ & Maximum transmit power at the BS & $30 \mathrm{dBm}$ \\
\hline$P_{\mathrm{V}}^{\mathrm{tot}}$ & Maximum transmit power at the UAV & $30 \mathrm{dBm}$ \\
\hline$N_{0}$ & Power spectral density of the noise & $-174 \mathrm{dBm} / \mathrm{Hz}$ \\
\hline$f_{c}$ & Carrier frequency & $5 \mathrm{GHz}$ \\
\hline$W_{\mathrm{U}}$ & Channel bandwidth of each UAV-UE link & $5 \mathrm{MHz}$ \\
\hline$W_{\mathrm{B}}$ & Channel bandwidth of the BS-UAV link & $K \times 5 \mathrm{MHz}$ \\
\hline$\alpha_{1}$ & Channel gain exponent for LoS path & 2 \\
\hline$\alpha_{2}$ & Channel gain exponent for NLoS path & 3.3 \\
\hline$\beta_{1}$ & $\begin{array}{l}\text { Channel gain at the reference distance } \\
\text { of 1 m for LoS path }\end{array}$ & $-46.43 \mathrm{~dB}$ \\
\hline$\beta_{2}$ & $\begin{array}{l}\text { Channel gain at the reference distance } \\
\text { of 1 m for NLoS path }\end{array}$ & $-56.43 \mathrm{~dB}$ \\
\hline$\lambda_{i}^{(0)}$ & Initial value of the Lagrangian multiplier & 1 \\
\hline$\rho^{(0)}$ & $\begin{array}{l}\text { Initial radius of the spherical region } \\
\text { in constraint (26c) }\end{array}$ & 50 \\
\hline$\kappa_{1}$ & $\begin{array}{l}\text { Step size for the radius reduction in } \\
\text { constraint (26c) }\end{array}$ & 0.9 \\
\hline$\epsilon_{\mathrm{t}}$ & Threshold for convergence of inner-loop & 0.01 \\
\hline$\epsilon_{\mathrm{T}}$ & Threshold for convergence of outer-loop & 0.01 \\
\hline$L_{\mathrm{t}, \mathrm{max}}$ & Maximum iteration number for inner-loop & 30 \\
\hline$L_{\mathrm{T}, \mathrm{max}}$ & Maximum iteration number for outer-loop & 10 \\
\hline & & \\
\hline
\end{tabular}

The proposed method is labeled by "LR". Besides, three benchmark schemes are defined for performance comparison, namely "ES", "CENTER" and "FREE", respectively.

- ES: This scheme performs an exhaustive search over a 3-D lattice with 5 meter spacing, which is defined as an upper bound on the minimum communication capacity for the UAV relay system. In each lattice, the optimal transmit powers given in Theorem 2 are adopted at the $\mathrm{BS}$ and the UAV relay. The candidate coordinates which achieve the maximum communication capacity for the actual channel environment are chosen as the optimal solution for UAV positioning.

- CENTER: The position of the UAV relay is set to be the center of the area with a height $H$ meters, and optimal transmit powers are adopted.

- FREE: A 2-D joint positing and power allocation problem with a fixed height $H$ is considered without geographic information. This problem is iteratively solved in a similar manner with Lines 4-10 in Algorithm 2.

\section{B. Simulation Results}

First, we provide a demonstration for the optimization process of the UAV positioning in Fig. 4, where 10 UEs are randomly distributed in the areas without buildings. The star labeled "start" is the initial position of the UAV, and the star 


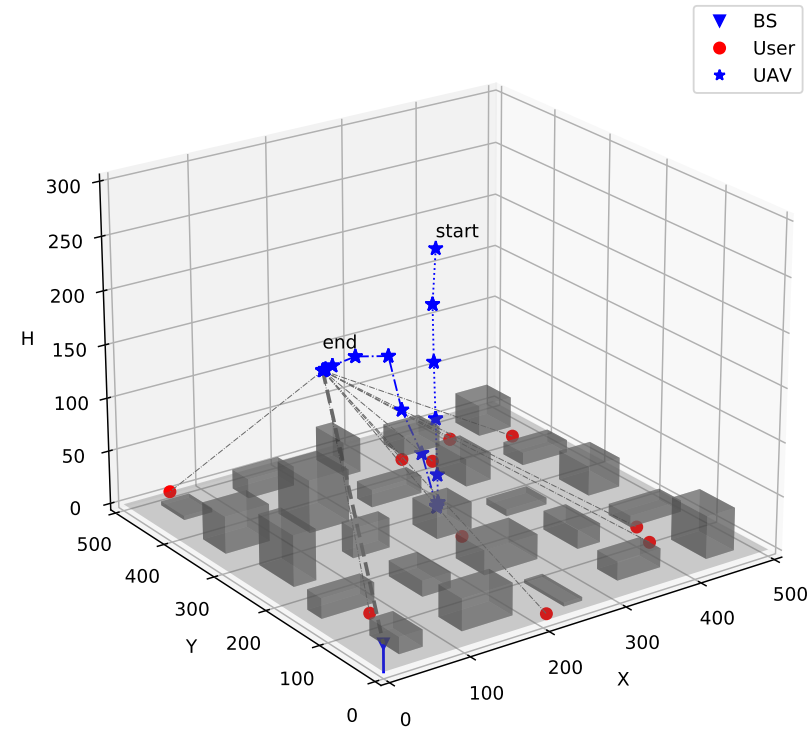

Fig. 4. Demonstration for UAV positioning with 10 UEs.

labeled "end" is the final position. Other stars represent the positions during the iteration. Different line types are used to distinguish the steps of outer-loops. It can be observed that the solution converges within three outer iterations. At the beginning of the iteration, with small initial Lagrangian multipliers, the UAV relay moves toward the ideal position without considering the existence of building blockages. As we can see, the UAV tends to decrease its height so as to get close to the ideal position. As the first outer-loop ends, the violation of blockage constraints leads to larger Lagrangian multipliers. Then, in the following outer-loop, the blockage constraints make sense and the UAV has to increase its height as well as adjust its horizontal position to avoid blockages. Finally, the position of the UAV relay is located outside blocked regions.

Fig. 5 demonstrates the convergence of the proposed Algorithm 2 versus the number of outer-loop iterations for different numbers of UEs. As can be observed, the gap between the objective value of Lagrangian problem (15) $q_{\mathrm{U}}$ and the objective value of original problem (12) $q_{\mathrm{B}}$ decreases with the iteration and converges within 5 iterations for all settings. The convergence of the outer-loop iteration means that appropriate values of the Lagrangian multipliers are obtained to ensure the UAV relay out of the blocked regions.

In Fig. 6, we evaluate the convergence of the last innerloop iteration of Algorithm 2 for different numbers of UEs. For comparison, the results of "ES" scheme are taken as upper bounds. It can be observed that the proposed method converges to a value close to the upper bound within 15 iterations for all settings. As the number of UEs increases, the gap increases slightly ( $0.25 \mathrm{Mbps}$ for $1 \mathrm{UE}, 1.56 \mathrm{Mbps}$ for 5 UEs, and $1.66 \mathrm{Mbps}$ for $10 \mathrm{UEs})$. This is because the unblocked region becomes narrow and decentralized with the increasing of the number of UEs, leading to multiple locally optimal locations. Even though, our algorithm achieves more than $95 \%$ capacity performance of the upper bound $(99.7 \%$ for

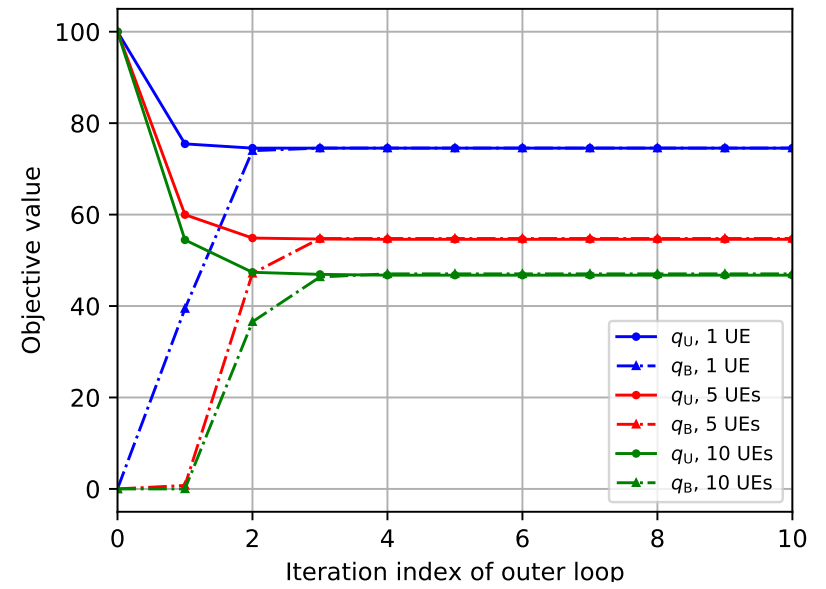

Fig. 5. Evaluation of the convergence of the outer-loop iteration of the proposed Algorithm 2 for different numbers of UEs.

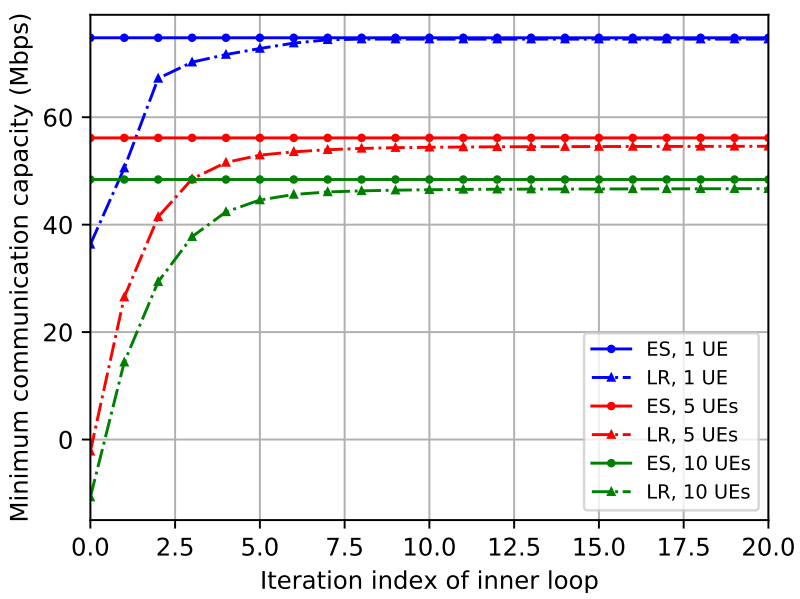

Fig. 6. Evaluation of the convergence of the inner-loop iteration of the proposed Algorithm 2 for different numbers of UEs.

1 UE, $97.2 \%$ for 5 UEs, and $96.6 \%$ for $10 \mathrm{UEs})$. The results confirm that with the optimized Lagrangian multipliers, the inner-loop for Lagrangian problem (15) can not only avoid blocked regions, but also obtain a near-optimal solution for original problem (12).

Fig. 7 compares the minimum communication capacities for different schemes versus the number of UEs. From the results, the performance of the proposed joint positioning and power allocation method aided by geographic information is very close to the performance upper bound given by "ES" scheme, and outperforms all other benchmark schemes. In addition, as the number of UEs increases, the minimum communication capacity decreases. The reason is as follows. On one hand, the maximum transmit power at the $\mathrm{BS} P_{\mathrm{B}}^{\text {tot }}$ and at the UAV relay $P_{\mathrm{V}}^{\text {tot }}$ are constant. As $K$ increases, the transmit power that can be potentially allocated to each UE is reduced, and thus leading to lower capacity. On the other hand, as $K$ increases, more blocked regions are involved. The UAV relay tends to be deployed at a higher altitude to avoid blockages, and thus leads to higher path loss and lower capacity.

Fig. 8 compares the minimum communication capacities 


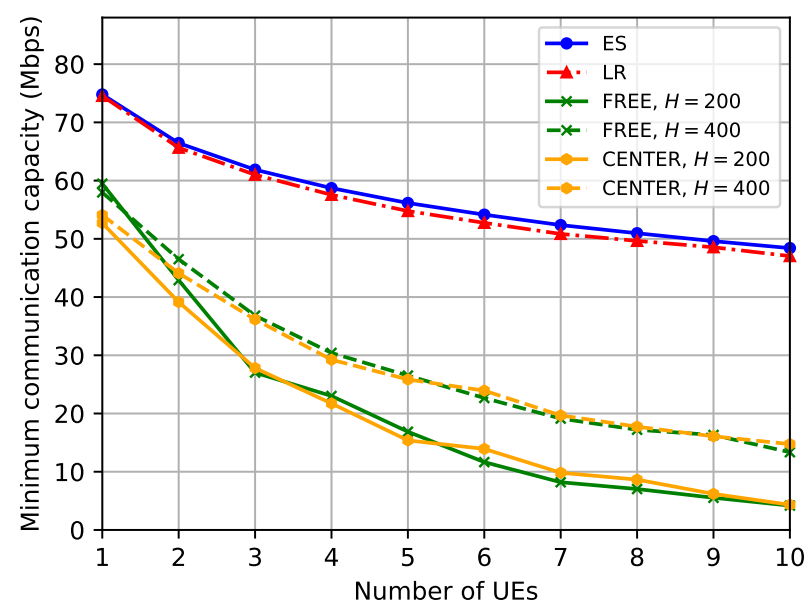

Fig. 7. Minimum communication capacities for different schemes versus the number of UEs.

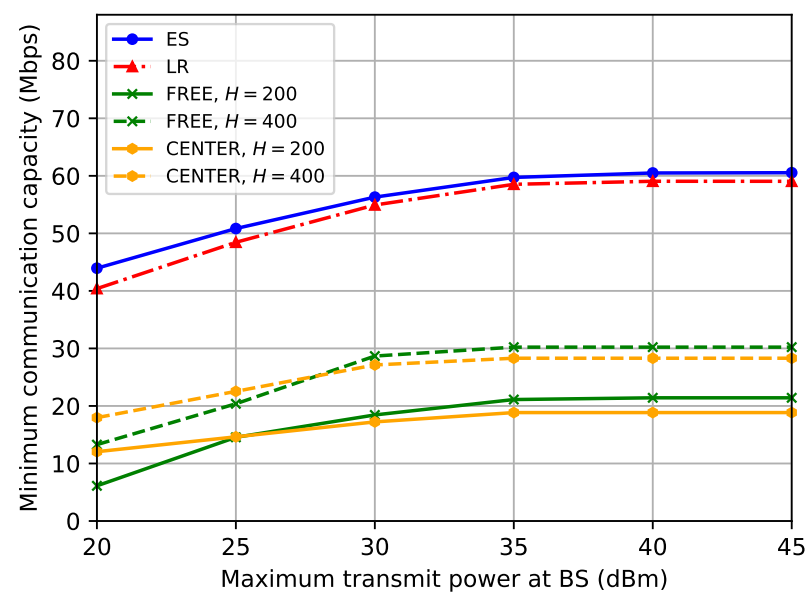

Fig. 8. Minimum communication capacities for different schemes versus BS transmit powers.

for different schemes versus the maximum transmit powers at the BS $\left(P_{\mathrm{B}}^{\text {tot }}\right)$, where the number of UEs is 5 . As can be observed again, the proposed method achieves a performance close to the upper bound and outperforms all other benchmark schemes. As $P_{\mathrm{B}}^{\text {tot }}$ increases, the minimum communication capacity of the proposed method improves, but with a decreased rate of improvement. The reason is as follows. When $P_{\mathrm{B}}^{\text {tot }}$ is small, the overall capacity is limited by the BS-UAV link. The UAV has to be positioned close to the BS. As $P_{\mathrm{B}}^{\text {tot }}$ increases, the limitation of the BS-UAV link is lightened, and the UAV positioning has more freedom to get a better performance. When $P_{\mathrm{B}}^{\text {tot }}$ is sufficiently large, the capacity of the BS-UAV link does not restrict the system performance, which, however, is limited by the UAV-UE links for fixed $P_{\mathrm{V}}^{\text {tot }}$. Therefore, the capacity does not increase as $P_{\mathrm{B}}^{\text {tot }}$ is larger than a certain threshold.

Fig. 9 compares the minimum communication capacities for different schemes versus the maximum transmit powers at the UAV $\left(P_{\mathrm{V}}^{\text {tot }}\right)$, where the number of UEs is 5. The proposed method still achieves a performance close to the upper bound and outperforms all the other benchmark schemes. When

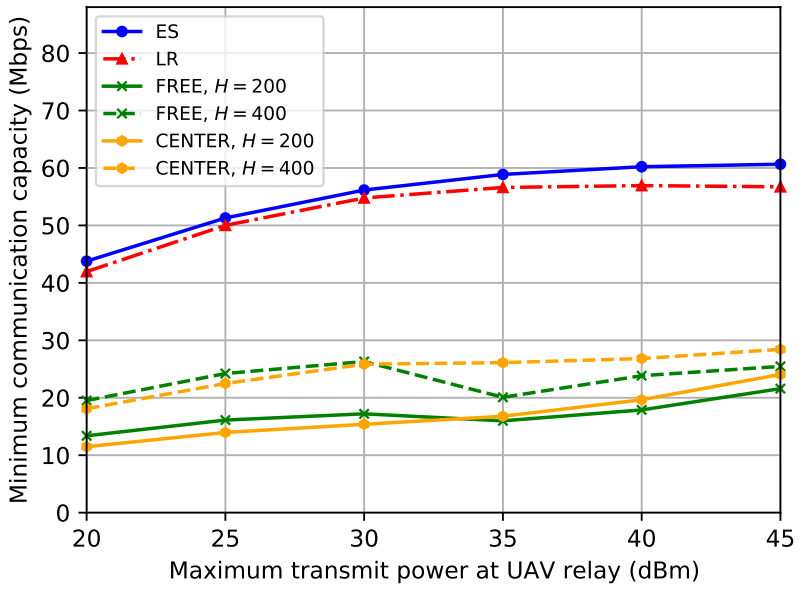

Fig. 9. Minimum communication capacities for different schemes versus UAV transmit powers.

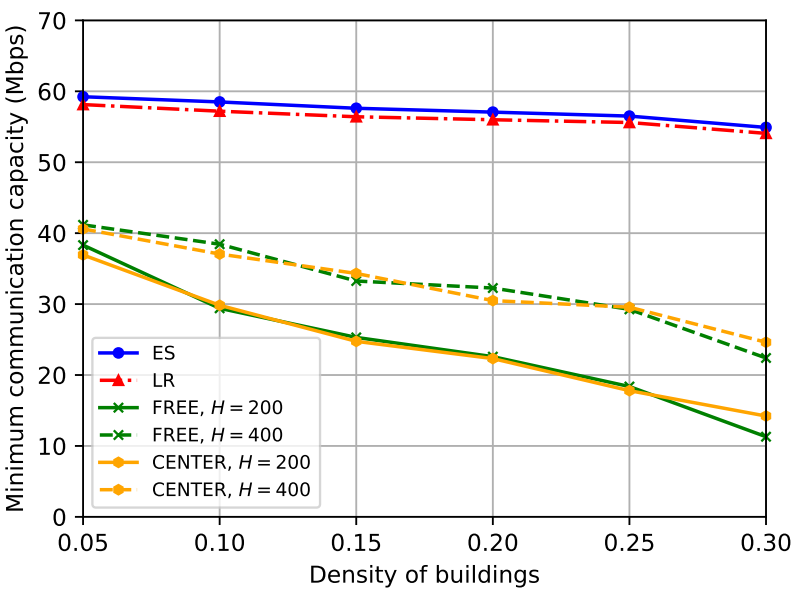

Fig. 10. Minimum communication capacities for different schemes versus building density.

$P_{\mathrm{V}}^{\text {tot }}$ is small, the communication capacity is limited by the UAV-UE links. As $P_{\mathrm{V}}^{\text {tot }}$ increases, the capacity of UAV-UE links becomes larger, thus leading to a better performance of the system. Note that the "FREE" scheme gets even worse performance as $P_{\mathrm{V}}^{\text {tot }}$ increases from $30 \mathrm{dBm}$ to $35 \mathrm{dBm}$. The reason is as follows. When $P_{\mathrm{V}}^{\text {tot }}$ increases from $30 \mathrm{dBm}$ to $35 \mathrm{dBm}$, the overall capacity is limited by the BS-UAV link. The UAV has to be positioned close to the BS, where UAV-UE links are more likely to be blocked by buildings. As $P_{\mathrm{V}}^{\text {tot }}$ continues to increase, even though the UAV-UE link is still blocked, the signal-to-noise ratio (SNR) of the UE receiver through NLoS link increases, thus leading to better performance. In contrast, the proposed method takes the blockage effect into account during the optimization and always obtains satisfactory performance for difference transmit powers. The results show the significance of the geographic information for the UAV relay systems.

Finally, we compare the minimum communication capacity for different schemes versus the density of buildings in Fig. 10, where the number of UEs is 5. The length and width of each building are random variables which follow a uniform 
distribution in a range which is properly determined to reach the desired building density. As can be observed, the minimum communication capacities for the considered schemes all decrease as the density of building increases. This is because denser buildings lead to more severe blockage effect. The UAV relay has to adjust its horizontal position and increase its altitude to avoid blockages. Even under this scenario, the proposed method still obtains a performance close to the upper bound and outperforms all other benchmark schemes. Besides, compared to "FREE" and "CENTER" benchmark schemes, the decreasing rate of the capacity obtained by the proposed method is lower, which suggests that the proposed method can efficiently get satisfying performance with the aid of geographic information for different building density circumstances.

\section{CONCLUSION}

In this paper, we proposed to employ a UAV relay to improve the minimum communication capacity from a ground BS to multiple UEs. Assisted by geographic information, the blockage effect caused by buildings was modeled. A joint optimization problem was formulated for the UAV positioning and power allocation under the constraints of link capacity, maximum transmit power, and blockage, to maximize the minimum communication capacity among all the UEs. To solve this non-convex problem, we proposed to leverage Lagrangian relaxation and performed a two-loop optimization framework. In the outer-loop, the Lagrange multipliers were adaptively updated to ensure the Lagrangian problem converge to the tightest upper bound on the original problem. In the innerloop, the Lagrangian problem was solved by applying the BCD technique, where UAV positioning and power allocation were alternately solved. Simulation results demonstrated that the proposed joint positioning and power allocation method aided by geographic information can closely approach a performance upper bound and outperforms two benchmark schemes in terms of the minimum communication capacity. The proposed blockage modeling method and the optimization framework have general significance for UAV positioning aided by geographic information.

\section{APPENDIX A}

\section{PROOF OF THEOREM 2}

First, to maximize the minimum communication capacity $R$, at least one of the transmit powers at the BS and UAV relay must reach the maximum possible value. The reason is as follows. Assume that the optimal transmit powers at the BS and UAV relay are both smaller than their maximum values, i.e., $P_{\mathrm{B}}^{\star}<P_{\mathrm{B}}^{\text {tot }}$ and $\sum_{k \in \mathcal{K}} P_{k}^{\star}<P_{\mathrm{V}}^{\text {tot }}$. Then, there exists a small positive value $\delta$ which ensures $(1+\delta) P_{\mathrm{B}}^{\star}$ and $\sum_{k \in \mathcal{K}}(1+$ $\delta) P_{k}^{\star}$ do not exceed the maximum values of their transmit powers. It can be verified that $\left((1+\delta) P_{\mathrm{B}}^{\star},\left\{(1+\delta) P_{k}^{\star}\right\}\right)$ yield a larger minimum communication capacity than $\left(P_{\mathrm{B}}^{\star},\left\{P_{k}^{\star}\right\}\right)$, which contradicts the assumption that $\left(P_{\mathrm{B}}^{\star},\left\{P_{k}^{\star}\right\}\right)$ is optimal.
Then, we show that with $\sum_{k \in \mathcal{K}} P_{k} \triangleq P_{\mathrm{V}} \leq P_{\mathrm{V}}^{\text {tot }}$, the optimal power allocation $\left\{P_{k}^{\star}\right\}$ to maximize the minimum communication capacity among all the UAV-UE link satisfies

$$
\eta_{1}^{(t)} P_{1}^{\star}=\eta_{2}^{(t)} P_{2}^{\star}=\ldots=\eta_{K}^{(t)} P_{K}^{\star} .
$$

The reason is as follows. Assume that (33) is not the optimal solution. Then at least one equality relationship in (33) is not true. By sorting $\left\{P_{k}^{\star}\right\}$ in ascending order, they follow

$$
\eta_{\pi_{1}}^{(t)} P_{\pi_{1}}^{\star}=\ldots=\eta_{\pi_{i}}^{(t)} P_{\pi_{i}}^{\star}<\eta_{\pi_{i+1}}^{(t)} P_{\pi_{i+1}}^{\star} \leq \ldots \leq \eta_{\pi_{K}}^{(t)} P_{\pi_{K}}^{\star},
$$

where $\left\{\pi_{k}\right\}$ are the indices after the proper permutation, and the first strict inequality occurs after the $\pi_{i}$-th term. Then, there exists a positive value $\Delta$ which ensures that

$$
\begin{aligned}
& \eta_{\pi_{1}}^{(t)}\left(P_{\pi_{1}}^{\star}+\Delta\right)=\ldots=\eta_{\pi_{i}}^{(t)}\left(P_{\pi_{i}}^{\star}+\Delta\right)= \\
& \eta_{\pi_{i+1}}^{(t)}\left(P_{\pi_{i+1}}^{\star}-i \Delta\right) \leq \ldots \leq \eta_{\pi_{K}}^{(t)} P_{\pi_{K}}^{\star},
\end{aligned}
$$

and yields a larger minimum communication capacity than $\left\{P_{k}^{\star}\right\}$, which contradicts the assumption that $\left\{P_{k}^{\star}\right\}$ is the optimal solution. Thus, (33) always holds for the optimal solution.

On one hand, the minimum communication capacity constrained by the BS-UAV link is

$$
\widetilde{R}=\frac{W_{\mathrm{B}}}{K} \log _{2}\left(1+\eta_{\mathrm{B}}^{(t)} P_{\mathrm{B}}\right) .
$$

On the other hand, combining $\sum_{k \in \mathcal{K}} P_{k}=P_{\mathrm{V}}$ with (33), the minimum communication capacity constrained by the UAVUE link is

$$
\widehat{R}=W_{\mathrm{U}} \log _{2}\left(1+\eta_{\mathrm{V}}^{(t)} P_{\mathrm{V}}\right)
$$

and the corresponding power allocation is $\widehat{P}_{k}=\eta_{\mathrm{V}}^{(t)} P_{\mathrm{V}} / \eta_{k}^{(t)}$.

When $\left(1+\eta_{\mathrm{B}}^{(t)} P_{\mathrm{B}}^{\text {tot }}\right)^{\frac{W_{\mathrm{B}}}{K}}<\left(1+\eta_{\mathrm{V}}^{(t)} P_{\mathrm{V}}^{\text {tot }}\right)^{W_{\mathrm{U}}}$, we have $\widetilde{R}<\widehat{R}$. Thus, $P_{\mathrm{B}}^{\star}=P_{\mathrm{B}}^{\text {tot }}$ maximizes the communication capacity of the BS-UAV link. Meanwhile, to avoid the waste of transmit power, $P_{\mathrm{V}}$ should be reduced to

$$
P_{\mathrm{V}}^{\star}=\left(\left(1+\eta_{\mathrm{B}}^{(t)} P_{\mathrm{B}}^{\mathrm{tot}}\right)^{\frac{W_{\mathrm{B}}}{K W_{\mathrm{U}}}}-1\right) / \eta_{\mathrm{V}}^{(t)} .
$$

Thus, the optimal power allocation at the UAV relay is

$$
P_{k}^{\star}=\left(\left(1+\eta_{\mathrm{B}}^{(t)} P_{\mathrm{B}}^{\mathrm{tot}}\right)^{\frac{W_{\mathrm{B}}}{K W_{\mathrm{U}}}}-1\right) / \eta_{k}^{(t)} \text {. }
$$

When $\left(1+\eta_{\mathrm{B}}^{(t)} P_{\mathrm{B}}^{\text {tot }}\right)^{\frac{W_{\mathrm{B}}}{K}} \geq\left(1+\eta_{\mathrm{V}}^{(t)} P_{\mathrm{V}}^{\text {tot }}\right)^{W_{\mathrm{U}}}$, we have $\widetilde{R} \geq \widehat{R}$. Thus, $P_{\mathrm{V}}^{\star}=P_{\mathrm{V}}^{\text {tot }}$ maximizes the communication capacity of the UAV-UE link. Meanwhile, to avoid the waste of transmit power, $P_{\mathrm{B}}$ should be reduced to

$$
P_{\mathrm{B}}^{\star}=\left(\left(1+\eta_{\mathrm{V}}^{(t)} P_{\mathrm{V}}^{\mathrm{tot}}\right)^{\frac{K W_{\mathrm{U}}}{W_{\mathrm{B}}}}-1\right) / \eta_{\mathrm{B}}^{(t)} .
$$

In addition, the optimal power allocation of the UAV relay is

$$
P_{k}^{\star}=\eta_{\mathrm{V}}^{(t)} P_{\mathrm{V}}^{\mathrm{tot}} / \eta_{k}^{(t)} .
$$

This completes the proof. 


\section{REFERENCES}

[1] L. Gupta, R. Jain, and G. Vaszkun, "Survey of important issues in UAV communication networks," IEEE Commun. Surveys Tuts., vol. 18, no. 2, pp. 1123-1152, second quarter 2016.

[2] Y. Zeng, Q. Wu, and R. Zhang, "Accessing from the sky: A tutorial on UAV communications for 5G and beyond," Proc. IEEE, vol. 107, no. 12, pp. 2327-2375, Dec. 2019.

[3] M. Mozaffari, W. Saad, M. Bennis, Y.-H. Nam, and M. Debbah, "A tutorial on UAVs for wireless networks: Applications, challenges, and open problems," IEEE Commun. Surveys Tuts., vol. 21, no. 3, pp. 2334 2360, third quarter 2019.

[4] A. Fotouhi, H. Qiang, M. Ding, M. Hassan, L. G. Giordano, A. GarciaRodriguez, and J. Yuan, "Survey on UAV cellular communications: Practical aspects, standardization advancements, regulation, and security challenges," IEEE Commun. Surveys Tuts., vol. 21, no. 4, pp. 3417-3442, fourth quarter 2019 .

[5] Z. Xiao, L. Zhu, and X.-G. Xia, "UAV communications with millimeterwave beamforming: Potentials, scenarios, and challenges," China Communications, vol. 17, no. 9, pp. 147-166, Sep. 2020.

[6] L. Song, Z. Han, B. Di, and H. Zhang, Aerial Access Networks: Integration of UAVs, HAPs, and Satellites. Cambridge, UK: Cambridge Univ. Press, 2021

[7] Q. Wu, Y. Zeng, and R. Zhang, "Joint trajectory and communication design for multi-UAV enabled wireless networks," IEEE Trans. Wireless Commun., vol. 17, no. 3, pp. 2109-2121, Mar. 2018.

[8] D. Xu, Y. Sun, D. W. K. Ng, and R. Schober, "Multiuser MISO UAV communications in uncertain environments with no-fly zones: Robust trajectory and resource allocation design," IEEE Trans. Commun., vol. 68 , no. 5 , pp. 3153-3172, May 2020.

[9] L. Zhu, J. Zhang, Z. Xiao, X. Cao, X.-G. Xia, and R. Schober, "Millimeter-wave full-duplex UAV relay: Joint positioning, beamforming, and power control," IEEE J. Select. Areas Commun., vol. 38, no. 9 , pp. 2057-2073, Sep. 2020.

[10] Y. Cai, F. Cui, Q. Shi, M. Zhao, and G. Y. Li, "Dual-UAV-enabled secure communications: Joint trajectory design and user scheduling," IEEE J. Select. Areas Commun., vol. 36, no. 9, pp. 1972-1985, Sep. 2018.

[11] X. Li, C. Pan, C. Zhang, C. He, and K. Wang, "Data rate maximization in UAV-assisted C-RAN," IEEE Wireless Communications Letters, vol. 9, no. 12, pp. 2163-2167, Dec. 2020.

[12] G. Zhang, Q. Wu, M. Cui, and R. Zhang, "Securing UAV communications via joint trajectory and power control," IEEE Trans. Wireless Commun., vol. 18, no. 2, pp. 1376-1389, Feb. 2019.

[13] H. Wang, J. Wang, G. Ding, J. Chen, Y. Li, and Z. Han, "Spectrum sharing planning for full-duplex UAV relaying systems with underlaid D2D communications," IEEE J. Select. Areas Commun., vol. 36, no. 9 , pp. 1986-1999, Sep. 2018.

[14] Y. Liu, K. Liu, J. Han, L. Zhu, Z. Xiao, and X.-G. Xia, "Resource allocation and 3-D placement for UAV-enabled energy-efficient IoT communications," IEEE Internet of Things Journal, vol. 8, no. 3, pp. 1322-1333, Feb. 2021.

[15] Z. Xiao, H. Dong, L. Bai, D. O. Wu, and X.-G. Xia, "Unmanned aerial vehicle base station (UAV-BS) deployment with millimeter-wave beamforming," IEEE Internet of Things Journal, vol. 7, no. 2, pp. 13361349, Feb. 2020.

[16] C. Zhang, L. Zhang, L. Zhu, T. Zhang, Z. Xiao, and X.-G. Xia, "3D deployment of multiple UAV-mounted base stations for UAV communications," IEEE Trans. Commun., vol. 69, no. 4, pp. 2473-2488, Apr. 2021.

[17] S. Zeng, H. Zhang, B. Di, and L. Song, "Trajectory optimization and resource allocation for OFDMA UAV relay networks," IEEE Trans. Wireless Commun., 2021 (Early access).

[18] T. Bai, R. Vaze, and R. W. Heath, "Analysis of blockage effects on urban cellular networks," IEEE Trans. Wireless Commun., vol. 13, no. 9, pp. 5070-5083, Sep. 2014.

[19] J. Chen, U. Mitra, and D. Gesbert, "3D urban UAV relay placement: Linear complexity algorithm and analysis," IEEE Trans. Wireless Commun., 2021 (Early access).

[20] Q. Hu, Y. Cai, A. Liu, G. Yu, and G. Y. Li, "Low-complexity joint resource allocation and trajectory design for UAV-aided relay networks with the segmented ray-tracing channel model," IEEE Trans. Wireless Commun., vol. 19, no. 9, pp. 6179-6195, Sep. 2020.

[21] J. Zhao, J. Liu, J. Jiang, and F. Gao, "Efficient deployment with geometric analysis for mmwave UAV communications," IEEE Wireless Communications Letters, vol. 9, no. 7, pp. 1115-1119, Jul. 2020
[22] J. F. Hughes, A. van Dam, M. McGuire, D. F. Sklar, J. D. Foley, S. Feiner, and K. Akeley, Computer Graphics: Principles and Practice, 3rd ed. Upper Saddle River, NJ: Addison-Wesley, 2013.

[23] S. Boyd and L. Vandenberghe, Convex optimization. Cambridge, UK: Cambridge Univ. Press, 2004.

[24] M. L. Fisher, "The Lagrangian relaxation method for solving integer programming problems," Management Science, vol. 27, no. 1, pp. 1-18, 1981.

[25] C. Xing, S. Wang, S. Chen, S. Ma, H. V. Poor, and L. Hanzo, "Matrixmonotonic optimization - part I: Single-variable optimization," IEEE Trans. Signal Processing, vol. 69, pp. 738-754, Feb. 2021.

[26] C. Xing, S. Wang, S. Chen, S. Ma, H. V. Poor, and L. Hanzo, "Matrixmonotonic optimization - part II: Multi-variable optimization," IEEE Trans. Signal Processing, vol. 69, pp. 179-194, Feb. 2021.

[27] Q. T. Dinh and M. Diehl, "Local convergence of sequential convex programming for nonconvex optimization," in Proc. Recent Advances in Optimization and its Applications in Engineering, Berlin, Heidelberg, 2010, pp. 93-102.

[28] L. Zhu, J. Zhang, Z. Xiao, X. Cao, D. O. Wu, and X.-G. Xia, "Millimeter-wave NOMA with user grouping, power allocation and hybrid beamforming," IEEE Trans. Wireless Commun., vol. 18, no. 11, pp. 5065-5079, Nov. 2019

[29] S. Diamond and S. Boyd, "CVXPY: A Python-embedded modeling language for convex optimization," Journal of Machine Learning Research, vol. 17 , no. 83 , pp. $1-5,2016$

[30] Z. Xiao, L. Zhu, J. Choi, P. Xia, and X.-G. Xia, "Joint power allocation and beamforming for non-orthogonal multiple access (NOMA) in 5G millimeter wave communications," IEEE Trans. Wireless Commun., vol. 17, no. 5, pp. 2961-2974, May 2018.

[31] D. P. Bertsekas, Nonlinear programming, 3rd ed. Belmont, MA, USA: Athena Scientific, 1999.

[32] A. Al-Hourani, S. Kandeepan, and S. Lardner, "Optimal LAP altitude for maximum coverage," IEEE Wireless Communications Letters, vol. 3 , no. 6, pp. 569-572, Dec. 2014.

[33] A. Al-Hourani, S. Kandeepan, and A. Jamalipour, "Modeling air-toground path loss for low altitude platforms in urban environments," in Proc. IEEE Glob. Commun. Conf., Austin, USA, Dec. 2014, pp. 28982904. 